\title{
A NANOSTRUCTURED IMPEDANCE MICROSENSOR FOR THE REAL-TIME MONITORING OF MACROMOLECULAR ASSEMBLY AND ELISA-ON-A-CHIP
}

\author{
F. Zang, X.Z. Fan, K. Gerasopoulos, L. Naves, J. Culver, and R. Ghodssi ${ }^{*}$ \\ University of Maryland, College Park, Maryland, USA
}

\begin{abstract}
This paper presents an impedimetric microsensor that monitors nano sensing probe functionalization in real-time to optimize on-chip enzyme-linked immunosorbent assays (ELISA). The self-assembly dynamics of Tobacco mosaic virus-like particle (VLP) bioreceptors were studied using continuous measurement of the electrical impedance between the interdigitated microelectrodes, which contributed to an optimized and reduced VLP assembly time from 18 hours to 3 hours. The impedance sensor with optimized VLP assembly effectively sensed antibody binding in on-chip ELISA which showed a $64 \%$ higher impedance change compared to the control experiment. These results combined highlight the significant potential of genetically modified VLPs as selective nanostructured probes for rapid immunoassays.
\end{abstract}

\section{INTRODUCTION}

Pathogenic microorganisms, including viruses, bacteria, fungi, parasites, etc., are major sources of diseases that threaten public health and food safety [1]. The rapid mutations of pathogens have degraded the efficacy of traditional therapies. A selective and sensitive diagnosis of pathogens is a crucial step in identifying the source of disease and form the right strategy for treatment. Immunoassays such as sandwiched enzyme-linked immunosorbent assays (ELISA) are among the most effective and widely used methods to sense specific types of pathogens. The sensing efficacy of immunoassays is largely determined by the density and binding affinity of the immobilized sensing probes. Currently, one major problem associated with the most commonly used antibody sensing probes in immunoassays is their low capture efficiency on the immobilized surface [2]. Because of this, surface functionalization is not maximized and the sensitivity of the immunoassay is not optimized. Besides, most surface functionalization procedures for immunoassays require several steps of chemical treatments on the surface. This prolongs the immunoassay, making it a time consuming and complex process. For these reasons, there is a need for development of sensing probes that are capable of functionalizing sensor surfaces rapidly and densely.

Tobacco mosaic virus (TMV) and its derivative virus-like particle (VLP) are emerging biomaterials that can serve as viable candidates for biosensing probes. TMV, a nanorod-structured plant virus with thousands of identical coat proteins on its outer surface, can be genetically modified to express functional residues. These include cysteines that facilitate self-assembly onto various substrates and peptides with high affinity to target molecules. Recent developments have led to the use of TMV as a biological nano-scaffold for surface decoration in energy applications or as a high affinity bioreceptor for selectivity enhanced sensing of chemical and biological molecules, respectively [3]. Owing to its high aspect ratio characteristic, the vertically self-assembled TMV is able to exhibit thousands of binding sites on its nanorod surface while maintaining a small footprint on a sensor surface. The latest research has successfully produced the genetically modified TMV coat proteins separately in bacteria cells. These coat proteins self-assembled into nanorod structured virus-like particles (VLPs) similar to TMVs in the absence of RNA strain with a much higher yield. VLPs are capable of creating dense functional surfaces in sensors for selective sensing of the biological binding events.
Previously, VLPs were used both as free floating particles in solution to capture explosives and immobilized receptors on transducers for antibody sensing [4, 5]. In the latter case, loss of VLPs due to repeated wetting and drying, combined with the long assembly time required to fully functionalize the surface, limited the device stability and throughput. Consequently, improving the procedure to perform immunoassays on-chip rapidly in liquid environments is required. Electrical impedance sensors provide suitable platforms for sensing macromolecular binding in liquid based on the changes in surface capacitance and resistance at low frequency due to the presence of particles between electrodes[6].

In this work, we leverage the benefits of impedance sensors such as good compatibility with liquid environments and high sensitivity to the surface capacitance/resistance change to monitor the nanoscale sensing probe functionalization in real-time and optimize on-chip ELISA. VLPs modified with cysteine groups and FLAG-tag peptides (VLP-FLAG) were used as sensing probes to anti-FLAG antibody to study the antigen-antibody interaction. The electrical impedance between the interdigitated microelectrodes is continuously monitored in the process of VLP-FLAG self-assembly in order to understand the dynamics of the VLP surface functionalization. The sensing efficacy of the VLP-FLAG functionalized impedance sensors with varying self-assembly time is studied by impedance shifts during the subsequent process of the on-chip ELISA. VLPs without binding peptides (VLP-1cys) are used in control experiments in the ELISA. The sensing efficacy and selectivity of VLP-FLAG and VLP-1cys are compared using independent impedimetric and colorimetric sensing methods.

\section{METHODS}

Impedance sensor designs and microfabrications

Impedance sensors with minimum feature size of $1 \mu \mathrm{m}$ were microfabricated and used for continuous monitoring of the VLP assembly dynamics and on-chip ELISA process.

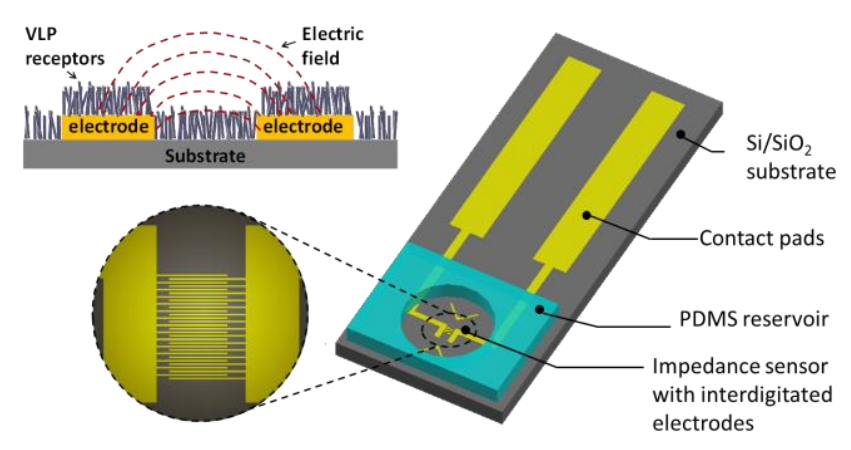

Figure 1: Schematic of the impedance sensor

Figure 1 shows a schematic of the impedance sensor which comprises gold interdigitated electrodes, contact pads and a PDMS reaction chamber. In the fabrication process, $\mathrm{Cr} / \mathrm{Au}(200 \AA / 1500 \AA)$ layers were first deposited on a $\mathrm{SiO}_{2} / \mathrm{Si}$ substrate using e-beam evaporation. The contact pads were defined by patterning Shipley 1813 positive photoresist via photolithography and etching the 
exposed $\mathrm{Cr} / \mathrm{Au}$ layer on the substrate. A $1.1 \mu \mathrm{m}$ thick 495 polymethyl methacrylate (PMMA) A10 layer was spin-coated and patterned via e-beam lithography. The subsequently deposited $\mathrm{Cr} / \mathrm{Au}(200 \AA / 1500 \AA)$ layer was lifted off to create interdigitated electrodes with $1 \mu \mathrm{m}$ finger width and spacing. The geometries of the interdigitated fingers were designed to be comparable to VLP dimensions in order to obtain a high sensitivity for monitoring the VLP sensing probe assembly dynamics and antibody binding events [6]. The effective sensing area of the interdigitated electrodes is $250 \mu \mathrm{m} \times 500 \mu \mathrm{m}$. A $3 \mathrm{~mm}$ thick Polydimethylsiloxane (PDMS) reaction chamber with a volume of $50 \mu \mathrm{l}$ was fabricated separately and bound onto the sensor substrate. During the experiments, an additional layer of PDMS is temporarily bound with the PDMS reaction chamber to seal the cavity, therefore, preventing the $50 \mu \mathrm{l}$ sample from evaporation and maintaining a constant analyte concentration. The reaction chamber and cover layer was used throughout the VLP self-assembly on the impedance sensor as well as the on-chip ELISA experiments.
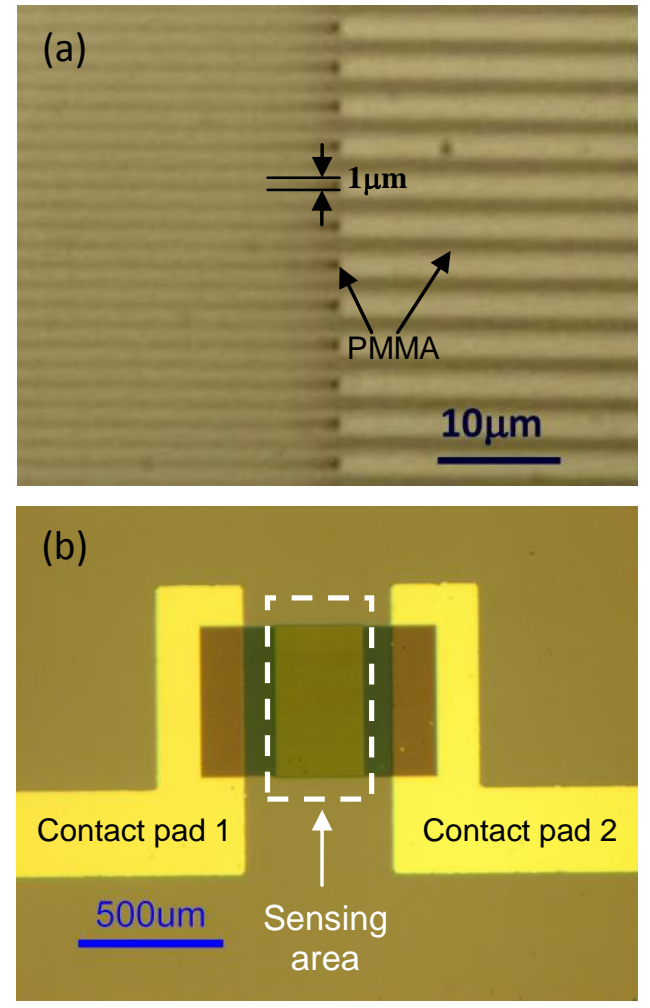

Figure 2: Optical images of (a) electrode features after e-beam lithography with PMMA e-beam resist and (b) the impedance sensor after fabrication

Figure 2a shows optical images of the 495 PMMA A10 resist after the e-beam lithography and the $\mathrm{O}_{2}$ plasma cleaning process. The plasma cleaning helps to remove PMMA residues from e-beam lithography and development, which contributes to clean interdigitated features. The patterned interdigitated electrodes after e-beam deposition of $\mathrm{Cr} / \mathrm{Au}$ and lift-off are shown in Figure $2 \mathrm{~b}$.

\section{VLP genetic modifications and surface functionalization}

The VLP sensing probes (VLP-FLAG) are synthetized by the helical arrangement of thousands of genetically modified and identical TMV coat proteins inside Escherichia coli bacterial cells.

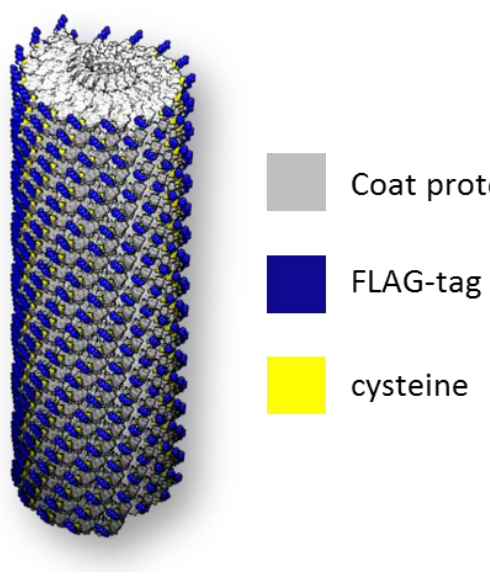

Figure 3: Schematic of a 3 dimensional segment of VLP with helical arrangement of genetically modified cysteine residues and FLAG-tag sequences on coat proteins

Each coat protein produced expresses a cysteine residue (marked in yellow in Figure 3) that promotes surface attachment and a FLAG-tag sequence (DYKDDDDK, marked in blue in Figure 3) that enables selective binding with the target anti-FLAG antibody. The genetic modification, culture and purification procedures were reported in detail previously [7]. The purified VLPs were diluted and suspended in deionized (DI) water at a previously characterized concentration of $0.2 \mathrm{mg} / \mathrm{ml}$. This VLP stock solution was used in the surface functionalization of the impedance sensors.

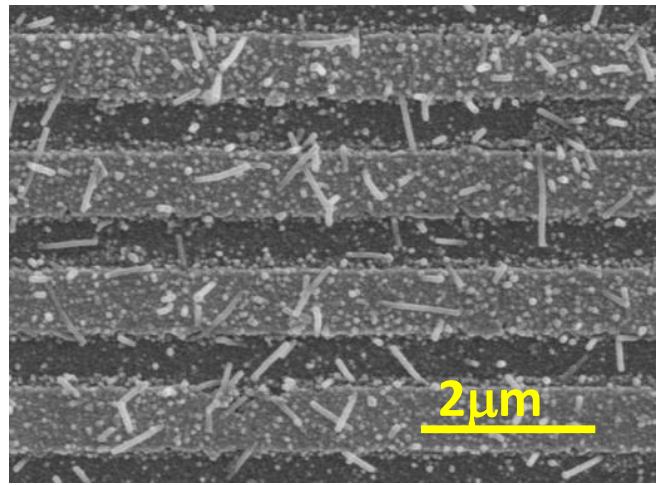

Figure 4: SEM images of interdigitated electrodes after VLP-FLAG assembly in deionized water

The VLP-FLAG sensing probes suspended in the stock solution were added in the PDMS reaction chamber to functionalize the impedance sensor. The cysteine residue exposed at the terminals of the VLP-FLAG nanorods contributed to vertical self-assembly of VLPs on the electrode area. The time of self-assembly was varied from 0 to 18 hours in the study. The VLP assembly for 18 hours was previously used as a standard assembly process to get a uniform VLP functional coating. The surface morphology of the VLP functional layer in the interdigitated electrode sensing area after 18 hours of assembly was studied by scanning electron microscopy (SEM). An electroless plating of nickel was performed before SEM imaging to promote the conductivity of the surface. The SEM image in Figure 4 shows the distribution of the VLP sensing probes on the sensing area of the impedance sensor after 18 hours of self-assembly. The 
self-assembled VLPs were observed both on the gold electrode and silicon oxide substrate.

The impedance between the interdigitated electrodes is monitored at a 15-minute interval using a potentiostat (VSP-300, Bio-Logic) to study the dynamics of the VLP assembly process. The real-time impedance monitoring is aimed at minimizing the VLP self-assembly time, shortening the duration of functionalization and ensuring the efficacy of antibody binding.

\section{Sandwiched ELISA on-chip with VLPs as sensing probes}

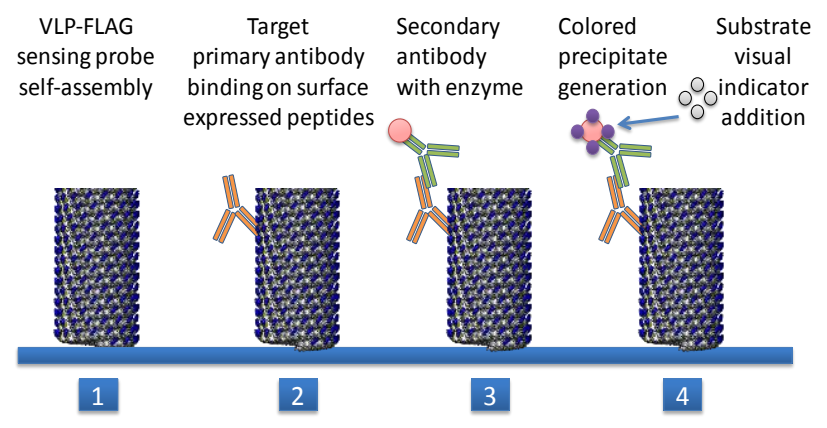

Figure 5: Sandwiched on-chip ELISA using VLP-FLAG

Sandwiched ELISA (Figure 5) was used as a model system to investigate the sensing efficacy of assembled VLP-FLAG sensing probes to anti-FLAG antibody targets. In the process, VLP-FLAG sensing probes were introduced in the reaction chamber and self-assembled on the impedance sensor surface. After washing with DI water, $5 \%$ milk in $1 \mathrm{X}$ Tris-buffered saline (TBS) buffer solution was introduced for 30 minutes to saturate the nonspecific bindings. After milk blocking and 3 times of $1 \mathrm{X}$ TBS washing, a 1:1000 dilution of primary anti-FLAG antibody produced in rabbit (Sigma-Aldrich) was introduced to bind with VLP sensing probes due to antigen-antibody interaction. A 1:5000 dilution of enzyme-linked anti-rabbit secondary antibodies (Sigma-Aldrich) were introduced in the reaction chamber subsequently after TBS and TBS/Tween washing steps. In the final step, the nitro-blue tetrazolium chloride and 5-bromo-4-chloro-3'-indolyphosphate p-toluidine salt (NBT/BCIP) substrates (Fischer Scientific) were added into the chamber and interacted with the enzyme on the secondary antibody, which produced dark-purple insoluble precipitates on the surface. The impedance of the sensor was continuously monitored during the ELISA experiments.

\section{RESULTS AND DISCUSSIONS}

The electrical impedance between the interdigitated electrodes is continuously monitored using the potentiostat while the frequency is swept from $7 \mathrm{MHz}$ to $1 \mathrm{~Hz}$ with a peak-to-peak potential of $50 \mathrm{mV}$. The electrical impedance is analyzed at the frequency of $100 \mathrm{~Hz}$ where the interfacial capacitance on the electrodes and the serial connected solution resistance are dominant in the electrical model [6].

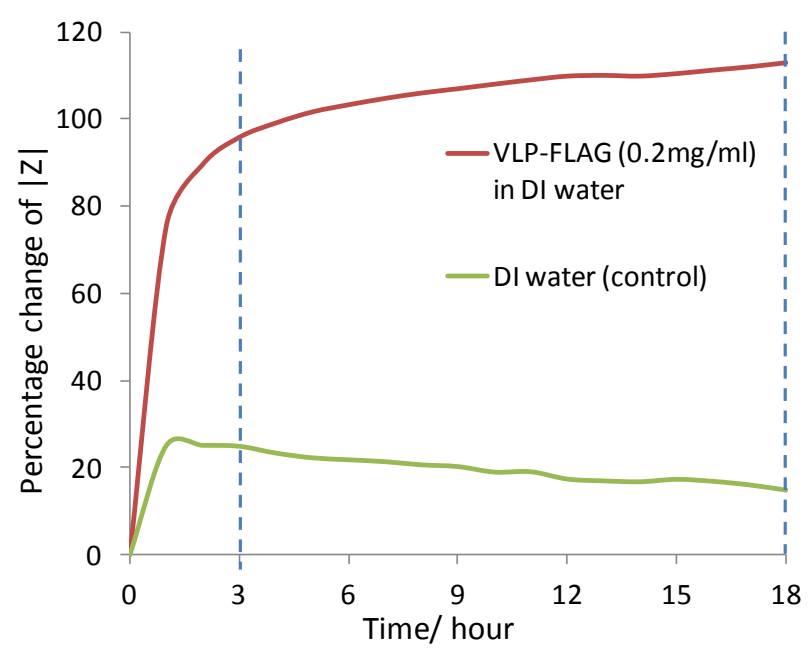

Figure 6: Percentage changes of impedance in the presence or absence of VLP-FLAG

VLP-FLAG sensing probes suspended in DI water $(0.2 \mathrm{mg} / \mathrm{ml})$ was added into the reaction chamber, and the impedance between interdigitated electrodes was monitored in real-time over the 18-hour period to study the VLP assembly dynamics. DI water, which acts as the solvent for VLP, was used in the parallel control experiment to generate a baseline impedance response. The percentage changes in the amplitude of impedance at the frequency of $100 \mathrm{~Hz}$ in the presence and absence of VLP probes are plotted in Figure 6. In the control experiment with DI water, the normalized impedance amplitude shows an increase of $25.1 \%$ in the first 1.5 hours and is stabilized at approximately $14.8 \%$ over the 18 -hour period. The normalized impedance of the sensor in the presence of VLP-FLAG increases dramatically in the first 3 hours by $95.7 \%$ after which it starts to saturate, gradually reaching up to $112.8 \%$ after 18 hours of assembly. The increase in the impedance amplitude can be a composite effect of the increase in the thickness of interface and the solution resistance between the electrodes due to the nonconductive VLP coating. These experimental results indicate the self-assembly of VLP-FLAGs on the impedance sensor saturates the surface within the first 3 hours. Therefore, the self-assembly procedure of VLP sensing probes can potentially be accelerated by shortening the assembly time while still maintaining a dense surface coverage. In the later experiments, the sensing efficacy of the impedance sensor with a 3-hour or 18-hour of VLP assembly is compared using on-chip ELISA.

The impedance sensors functionalized by 3-hour and 18-hour VLP-FLAG self-assembly or 18-hour of VLP-1cys self-assembly was used to study the sensing efficacy of VLP probes. VLP-1cys, expressing only cysteine residues without antibody binding peptide sequences, was used as a control probe. The impedance after each process during the ELISA (blocking, primary and secondary antibody binding and substrate introduction) was measured in $1 \mathrm{X}$ TBS buffer to normalize the background. 


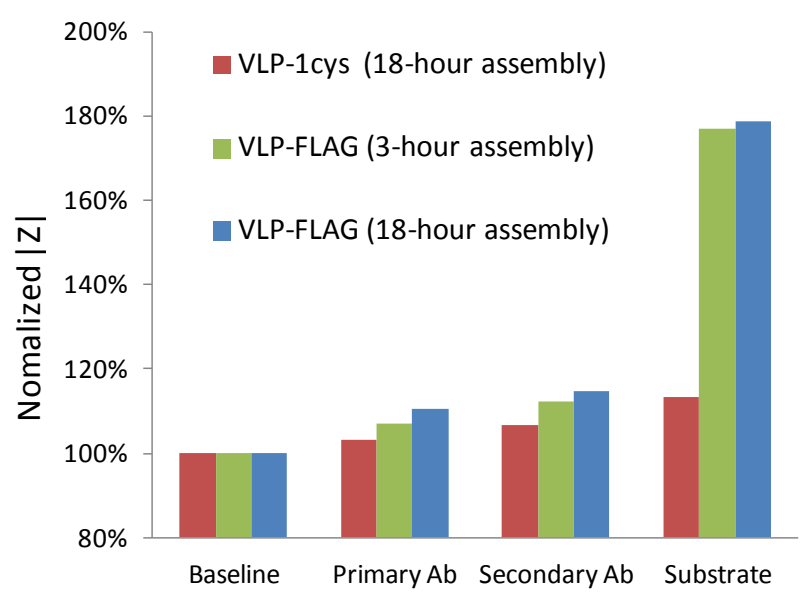

Figure 7: Normalized impedance of VLP-FLAG or unmodified VLP functionalized sensors during ELISA

Figure 7 shows the normalized impedance amplitude during ELISA experiments. The baseline for each experiment was acquired based on the measured impedance after 30 minutes of 5\% milk nonspecific blocking. The total normalized impedance for sensors functionalized for 3 hours and 18 hours using VLP-FLAG increased by $77.1 \%$ and $77.8 \%$, respectively, indicating a similar biosensing efficacy in immunoassay. The significant change in impedance is due to the formation of insoluble precipitates that cover the sensing area and alter the surface morphology. This change prevents the ion transfer to the electrode, and creates thicker interface capacitance; thus, both the real and imaginary parts of impedance between electrodes show significant increase. In comparison, when VLP-1cys control probes are used, the impedance change is only $13.3 \%$, which is attributed to non-specific binding. The distinguishing difference of impedance after ELISA between the VLP-FLAG and VLP-1cys assembled sensors verified the selectivity of the genetically modified VLPs to the target antibodies.

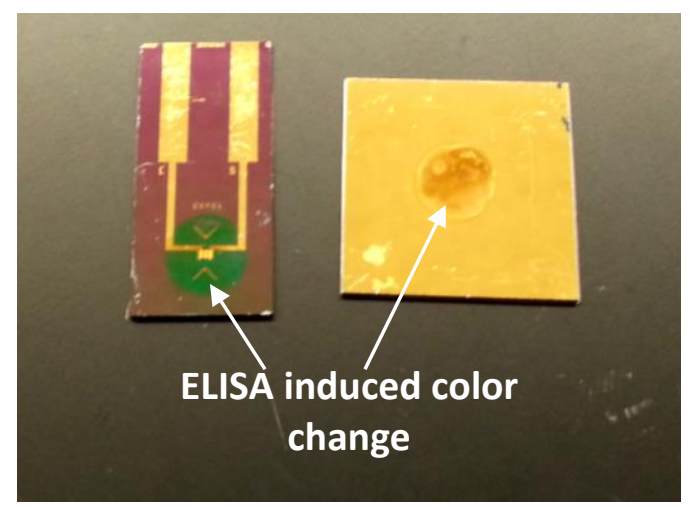

Figure 8: Optical image of (left) the impedance sensor and (right) bare gold chips with identical 3 hours of VLP-FLAG self-assembly after ELISA

The functionality of the VLP-FLAG as sensing probes in ELISA was independently validated by colorimetric experiments conducted in parallel with the impedimetric sensing experiments. Dark-purple colored precipitates due to the interaction between substrate and enzymes on the secondary antibodies were observed on impedance sensors and gold chips which were decorated with VLP-FLAG through self-assembly (Figure 8).

\section{SUMMARY}

Impedance sensors were developed to study the dynamics of biological sensing probe assembly on sensor surfaces and the impedimetric responses during immunoassays. The continuous impedance monitoring over 18 hours of VLP-FLAG assembly showed an optimized assembly time of 3 hours which saturated the impedance sensor surface. ELISA experiments validated the selectivity and sensing efficacy of the sensor surface functionalized by the VLP-FLAG sensing probes with 3 hours of self-assembly.

This work provided important self-assembly parameters of VLP sensing probes to reduce the functionalization time of transducer surfaces and accelerate immunoassays. It will contribute to the future development of programmable VLP-based biosensors for rapid macromolecule sensing.

\section{ACKNOWLEDGEMENTS}

The work was supported by the Army Research Office Biochemistry Program (Grant No. W911NF1110138) and National Science Foundation Nanomanufacturing Program (Grant No. NSF-CMMI 0927693). The authors would like to acknowledge the staff in Maryland Nanocenter for the support in fabrication process.

\section{REFERENCES}

[1] L. Yang and R. Bashir, "Electrical/electrochemical impedance for rapid detection of foodborne pathogenic bacteria," Biotechnology Advances, 26, 135-150, (2008).

[2] M. Varshney, Y.B. Li, B. Srinivasan, and S. Tung, "A label-free, microfluidics and interdigitated array microelectrode-based impedance biosensor in combination with nanoparticles immunoseparation for detection of Escherichia coli O157 : H7 in food samples," Sensors and Actuators B-Chemical, 128, 99-107, (2007).

[3] X.Z. Fan, E. Pomerantseva, M. Gnerlich, A. Brown, K. Gerasopoulos, M. McCarthy, J. Culver, and R. Ghodssi, "Tobacco mosaic virus: A biological building block for micro/nano/bio systems," Journal of Vacuum Science \& Technology A, 31, (2013).

[4] F. Zang, X.Z. Fan, K.D. Gerasopoulos, H. Ben-Yoav, A.D. Brown, J.N. Culver, and R. Ghodssi, "Scale-down effects: Towards miniaturization of an electrochemical sensor using biomolecules," in IEEE Sensors 2013, Baltimore, USA, 2013, pp. 1-4.

[5] X.Z. Fan, L. Naves, N. P. Siwak, A.D. Brown, J. N. Culver, and R. Ghodssi, "A Novel Virus-like-particle (VLP) Bioreceptor Coated Optical Disk Resonator for Biosensing,," presented at the 2013 MRS Spring Meeting, San Francisco, CA, USA, (2013).

[6] P. Van Gerwen, W. Laureyn, W. Laureys, G. Huyberechts, M.O. De Beeck, K. Baert, J. Suls, W. Sansen, P. Jacobs, L. Hermans, and R. Mertens, "Nanoscaled interdigitated electrode arrays for biochemical sensors," Sensors and Actuators B-Chemical, 49, 73-80, (1998).

[7] A.D. Brown, L. Naves, X. Wang, R. Ghodssi, and J.N. Culver, "Carboxylate-Directed In Vivo Assembly of Virus-like Nanorods and Tubes for the Display of Functional Peptides and Residues," Biomacromolecules, 14, 3123-3129, (2013).

\section{CONTACT}

*R. Ghodssi, tel: +1-301-405-8158; ghodssi@umd.edu 